\title{
Random ergodic theorems for sub-Markovian operators
}

Abstract. We prove a random ergodic theorem for positive weak*-continuous contractions on $L^{\infty}$. This theorem generalizes a random ergodic theorem of Ryll-Nar dzewski [15] and some results of Nawrotzki [11].

In the classical random ergodic theory the average behavior of measurepreserving transformations chosen at random from a set $\Phi$ is studied. One of the problems is the structure of the limit function. This problem was considered by Kakutani [8], Ryll-Nardzewski [15] and Gładysz [6], [7]; the operator-theoretical generalizations were given by Cairoli [2] The case of a finite phase space was considered by Nawrotzki [11].

In [2] Cairoli obtained an extension of Ryll-Nardzewski random ergodic theorem by considering a measurable family of positive contractions on $L_{1}$ having a strictly positive invariant function. The aim of the present paper is to give a natural extension of Ryll-Nardzewski theorem [15] and of the main results of Nawrotzki [11] by considering a measurable family $\mathscr{P}=\left\{P_{s}: s \in S\right\}$ of sub-Markovian operators, i.e. positive weak*-continuous contractions on $L_{\infty}$.

Section 1 is preliminary. Haring a measurable family $\mathscr{P}=\left\{P_{s}: s \in \mathbb{S}\right\}$ of sub-Markovian operators we define a sub-Markovian operator $U$ (which corresponds to the classical skew product transformation). Section 2 is devoted to the study of invariant functions and measures for the operator $U$. In Theorem 2.4. it is proved that the $U$-invariant functions do not depend essentially on random parameters on the conservative part of $U$. The analogous result for the $U$-invariant measures is given in Theorem 2.6. The method of proof of this theorem can also be applied to non-positive operators (see [17]). In Section 3 we state a random version of the individual and strong ergodic theorems together with the identification of the limit function (Theorems 3.4 and 3.6). Finally, Section 4 contains an example connected with Theorem 3.6. It is shown that given a two-sided Bernoulli shift there exists an integrable function $f$ with the property that the ergodic averages conditioned on the future are divergent almost 
everywhere. We note that a similar example was exhibited by Burkholder in [1] but it is not sufficient for our purposes.

The results in Sections 1-3 form a part of author's doctoral thesis written under the supervision of Professor S. Gładysz. The author wishes to thank Professor S. Gladysz and Professor O. Ryll-Nardzewski for their helpful comments and stimulating conversations concerning the contents of this paper.

1. Preliminaries. Let $(X, \mathscr{A}, \mu)$ be a $\sigma$-finite measure space and let; $(S, \mathscr{B}, \lambda)$ be a probability space. By $\left(S^{*}, \mathscr{B}^{*}, \lambda^{*}\right)$ wo denote a countablo product of $(S, \mathscr{B}, \lambda): \quad S^{*}=S_{1} \times S_{2} \times \ldots, \mathscr{B}^{*}=\mathscr{B}_{1} \times \mathscr{B}_{2} \times \ldots, \lambda^{*}=\lambda_{1} \times$ $\times \lambda_{2} \times \ldots$, where $S_{i}=S, \mathscr{B}_{i}=\mathscr{B}, \lambda_{i}=\lambda$ for $i=1,2, \ldots$ By $\xi$ we shall denote the shift transformation on $S^{*}$, i.e. $\left(s_{1}, s_{2}, \ldots\right) \xi=\left(s_{2}, s_{3}, \ldots\right)$ for each $\left(s_{1}, s_{2}, \ldots\right) \in S^{*}$.

In this paper only real measurable functions are considered and they are denoted by $f, g, h$. For each $1 \leqslant p \leqslant \infty$ by $L_{p}(X, \mathscr{A}, \mu)=L_{p}(\mu)$ wo denote the usual Banach spaces and $L_{p}^{+}(\mu)$ denotes a cone consisting of all nonnegative elements from $L_{p}(\mu)$. All inequalitios and limit operations appearing in this paper are understood almost everywhere, unless stated otherwise. Furthermore, we write $\lim f_{n}=f$ on $A$ if $\lim f_{n}(x)=f(x)$ for $\mu$-almost all $x \in A \subseteq X$.

The analogous notation is applied to the measure space $\left(X \times S^{*}\right.$ $\left.\mathscr{A} \times \mathscr{B}^{*}, \mu \times \lambda^{*}\right)$ and to functions defined on $X \times \mathcal{S}^{*}$. If we wish to regard $f\left(x ; s^{*}\right)$ as a function of $x$ defined on $X$ for an $s^{*}$ arbitrary fixed in $S^{*}$, we shall write $f_{\left(s^{*}\right)}(x)$ for $f\left(x ; s^{*}\right)$.

Now, let $\mathscr{P}=\left\{P_{s}: s \in \mathscr{S}\right\}$ be a family of sub-Markovian operators on $L_{\infty}(\mu)$, i.e. for each $s \in \mathbb{S} P_{8}$ is a linear operator on $L_{\infty}(\mu)$ (whose value on $f \in L_{\infty}(\mu)$ is denoted by $P_{s} f$ ) with the following properties:

(i) $P_{s} 1 \leqslant 1$ on $X$,

(ii) if $f \in L_{\infty}^{+}(\mu)$, then $P_{s} f \in L_{\infty}^{+}(\mu)$,

(iii) if $\lim _{n \rightarrow \infty} 1_{A_{n}}=0$ on $X\left(A_{n} \in \mathscr{A}\right)$, then $\lim _{n \rightarrow \infty} P_{s} 1_{A_{n}}=0$ on $X$.

The conditions (i)-(iii) are equivalent to the statement that each operator $P_{s} \in \mathscr{P}$ is a positive weak ${ }^{*}$-continuous contruction on $L_{\infty}(\mu)$. Therefore, each operator $P_{s} \in \mathscr{P}$ is an adjoint to a positive contraction on $L_{1}(\mu)$, which is denoted by the same symbol $P_{s}$, but is written to the right of its variable. So,

$$
\int_{X} f P_{s} \cdot g d \mu=\int_{X} f \cdot P_{s} g d \mu
$$

for $f \in L_{1}(\mu), g \in L_{\infty}(\mu)$.

1.1. Definrmion. The family $\mathscr{P}=\left\{P_{\theta}: s \in S\right\}$ of sub-Markovian operators on $L_{\infty}(\mu)$ is called measurable if for every $A \in \mathscr{A}$ there exists a $\mathscr{A} \times \mathscr{B}$. measurable function $g(x, s)$ and a set $S^{\prime} \in \mathscr{B}$ with $\lambda\left(S^{\prime}\right)=1$ such that for every $s \in S^{\prime} P_{s}^{\prime} 1_{A}(x)=g_{(s)}(x)$ on $X$.

Now, throughout the paper we fix a triple consisting of a $\sigma$-finite measure space $(X, \mathscr{A}, \mu)$, a probability space $(\mathcal{S}, \mathscr{B}, \lambda)$ and a measurable farnily $\mathscr{P}=\left\{P_{s}: s \in S\right\}$ of sub-Markovian operators on $L_{\infty}(\mu)$.

1.2. Timma. Fror every $f \in I_{\infty}\left(\mu \times \lambda^{*}\right)$ there exists a $g \in I_{\infty}\left(\mu \times \lambda^{*}\right)$ and a set $S^{* \prime} \in \mathscr{B}^{*}$ with $\lambda^{*}\left(S^{* \prime \prime}\right)=1$. such that for each $s^{*}=\left(s_{1}, s_{2}, \ldots\right) \in S^{* \prime}$

$$
\left(P_{B_{1}} f_{\left(s_{2}, B_{3}, \ldots\right)}\right)(x)=g_{\left(s^{*}\right)}(x) \text { on } X \text {. }
$$

The function $g\left(x ; s^{*}\right)$ is determinod uniquely $\bmod \left(\mu \times \lambda^{*}\right)$.

Proof. Without loss of generality we may assume that the measure $\mu$ is finite. First, let ns consider a function $f \in L_{\infty}\left(\mu \times \lambda^{*}\right)$ of the form

$$
f\left(x ; s^{*}\right)=\sum_{i, j=1}^{k} a_{i j} 1_{A_{i}}(x) 1_{B_{j}^{*}}\left(s^{*}\right),
$$

where $A_{1}, \ldots, A_{k}\left[B_{1}, \ldots, B_{l k}\right]$ is a partition of $X\left[S^{*}\right]$ into mutually disjoint $\mathscr{A} /$-measurable $\left[\mathscr{S}{ }^{*}\right.$-measurable] sets and $a_{i j}$ are some real numbers. By 1.1 there exist functions $g_{i}\left(x ; s_{1}\right) \in L_{\infty}\left(\mu \times \lambda_{1}\right)$ and a set $S_{1}^{\prime} \in \mathscr{B}_{1}$ with $\lambda_{1}\left(S_{1}^{\prime}\right)=1$ such that for every $s_{1} \in S_{1}^{\prime}$

$$
\left(g_{i}\right)_{\left(s_{1}\right)}(x)=P_{B_{1}} \mathcal{I}_{A_{i}}(x) \quad \text { on } \quad X
$$

$(i=1, \ldots, k)$. Since for each $s^{*}=\left(s_{1}, s_{2}, \ldots\right) \in S_{1}^{\prime} \times S_{2} \times S_{3} \times \ldots$, we have

$$
\left(P_{B_{1}} f_{\left(s^{*} \xi\right.}\right)(x)=\sum_{i, j=1}^{k} a_{i j} P_{s_{1}} 1_{A_{i}}(x) 1_{B_{j}^{*}}\left(s^{*} \xi\right),
$$

wo infer that the function

$$
g\left(x ; s^{*}\right)=\sum_{i, j=1}^{k} a_{i j} g_{i}\left(x ; s_{1}\right) 1_{B_{j}^{*}}\left(s^{*} \xi\right)
$$

satisfies (1).

Now, for an arbitrary function $f \in I_{\infty}\left(\mu \times \lambda^{*}\right)$ there exists a sequence $f_{n}\left(x ; s^{*}\right)$ of functions of the form (2) such that $\lim f_{n}\left(x ; s^{*}\right)=f\left(x ; s^{*}\right)$ on $X \times S^{*}$ and that $\left|f_{n}\left(x ; s^{*}\right)\right| \leqslant M<\infty$ on $X \times S^{*}$. Therefore there exists an sut $S^{* \prime} \in \mathscr{B}^{*}$ with $\lambda^{*}\left(S^{* \prime}\right)=1$ such that for every $8^{*} \in S^{* \prime}$

and that

$$
\lim _{n \rightarrow \infty}\left(f_{n}\right)_{\left(\theta^{*} \xi\right)}(x)=f_{\left(8^{*}\right)}(x) \quad \text { on } \quad X
$$

$$
\left|\left(f_{n}\right)_{\left(\theta^{*} \xi\right)}(\infty)\right| \leqslant M \text { on } \quad X \text {. }
$$

Hence, for each $s^{*} \in S^{* \prime} \lim \left(f_{n}\right)_{\left(s^{*} \xi\right)}=f_{\left(s^{*} \xi\right)}$ in the weak $k^{*}$-topology of $L_{\infty}(\mu)$ and, by the weak ${ }^{*}$-continuity of $P_{s}$,

(4) $\quad \lim _{n \rightarrow \infty} P_{s_{1}}\left(f_{n}\right)_{\left(s^{*} \xi\right)}=P_{s_{1}} f_{\left(s^{*} \xi\right)}$ in the weak-topology of $L_{\infty}(\mu)$. 
As we have seen, there exist $g_{n}\left(x ; s^{*}\right) \in L_{\infty}\left(\mu \times \lambda^{*}\right)$ and a set $\mathcal{S}^{* \prime \prime} \in \mathscr{B}^{*}$ with $\lambda^{*}\left(S^{* \prime \prime}\right)=1$ such that for each $s^{*}=\left(s_{1}, s_{2}, \ldots\right) \in S^{* \prime \prime}$ and each $n=1,2, \ldots$

(5) $\quad\left(P_{s_{1}}\left(f_{n}\right)_{\left(g^{*} \xi\right)}\right)(x)=\left(g_{n}\right)_{\left(s^{*}\right)}(x)$ on $X$.

Moreover, since each operator $P_{s} \in \mathscr{P}$ is sub-Markovian, the inequality (3) implies

$$
\left|g_{n}\left(x ; s^{*}\right)\right| \leqslant M \quad \text { on } \quad X \times \mathbb{S}^{*} .
$$

Now, we shall prove that the sequence $g_{n}$ is fundamental in the weak*-topology of $L_{\infty}\left(\mu \times \lambda^{*}\right)$. In view of $(6)$ it is sufficient to check that for every function $h\left(x ; s^{*}\right) \in L_{1}\left(\mu \times \lambda^{*}\right)$ of the form $h\left(x ; s^{*}\right)=h_{1}(x) h_{2}\left(s^{*}\right)$ $\lim _{n \rightarrow \infty} \int_{X \times S^{*}} \int_{S^{*}} d\left(\mu \times \lambda^{*}\right)$ exists. It follows from (4) and (5) that for each $s^{*} \in S^{* \prime \prime}$ $\lim _{n \rightarrow \infty} \int_{X} h_{1}(x) g_{n}\left(x ; s^{*}\right) d \mu(x)$

exists and, by (6), that

$$
\left|\int_{X} h_{1}(x) g_{n}\left(x ; s^{*}\right) d \mu(x)\right| \leqslant M \cdot\left\|h_{1}\right\|_{L_{1}(\mu)} .
$$

Therefore $\lim _{n \rightarrow \infty} \iint_{X \times S^{*}} h g_{n} d\left(\mu \times \lambda^{*}\right)$ exists by the Lebesgue dominated conver gence theorem. Since the weak*-fundamental sequences are weak*-convergent, there exists a function $g\left(x ; s^{*}\right) \in L_{\infty}\left(\mu \times \lambda^{*}\right)$ such that $\lim _{n \rightarrow \infty} g_{n}=g$ in the weakk $k^{*}$-topology of $L_{\infty}\left(\mu \times \lambda^{*}\right)$. It follows from (4) and (5) that $g$ satisfies (1), which proves the lemma.

Now, let $f \in L_{\infty}\left(\mu \times \lambda^{*}\right)$ and let $g$ be a function determined by Lemma 1.2. We define a linear operator $U$ on $L_{\infty}\left(\mu \times \lambda^{*}\right)$ by

$$
\sigma f=g \text {. }
$$

It is easy to check that $U$ is a sub-Markovian operator on $L_{\infty}\left(\mu \times \lambda^{*}\right)$. In particular, $U$ is an adjoint to some positive contraction on $I_{1}\left(\mu \times \lambda^{*}\right)$, which will be denoted by the same letter $U$, but will be writton to the right of its variable. Hence

$$
\iint_{X \times S^{*}} f U \cdot g d\left(\mu \times \lambda^{*}\right)=\iint_{X \times S^{*}} f \cdot U g d\left(\mu \times \lambda^{*}\right)
$$

for $f \in L_{1}\left(\mu \times \lambda^{*}\right), g \in L_{\infty}\left(\mu \times \lambda^{*}\right)$.

The following lemma, which is easy to prove, describes the action of the iterates of the operator $U$.

1.3. Luma. Let $f \in I_{\infty}\left(\mu \times \lambda^{*}\right)$. Then there exists a set $S^{* \prime} \in \mathbb{S}^{*}$ with $\lambda^{*}\left(S^{* \prime}\right)=1$ such that for every $s^{*}=\left(s_{1}, s_{2}, \ldots\right) \in S^{* \prime}$ and every $n=1,2, \ldots$

$$
\left(U^{n} f\right)_{\left(s^{*}\right)}(x)=P_{s_{1}} P_{s_{2}} \ldots P_{s_{n}} f_{\left(s_{n+1}, s_{n+2}, \ldots\right)}(x) \text { on } \quad X \text {. }
$$

Now, for every $n=0,1,2, \ldots$ let $\nexists^{6_{n}}$ denotes a sub-Markovian operator on $L_{\infty}\left(\mu \times \lambda^{*}\right)$ which sends to every function $f \in L_{\infty}\left(\mu \times \lambda^{*}\right)$ the conditional expectation $\mathbb{H}^{6} n f$ with respect to the sub- $\sigma$-field

$$
\mathscr{C}_{n}=\mathscr{A} \times \mathscr{B}_{1} \times \ldots \times \mathscr{B}_{n} \times\left\{\varnothing, s_{n+1}\right\} \times\left\{\varnothing, s_{n+2}\right\} \times \ldots
$$

We have

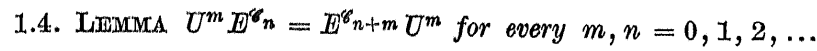

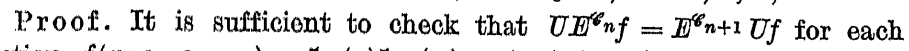
function $f\left(x ; s_{1}, s_{2}, \ldots\right)=1_{A}(\infty) 1_{B_{1}}\left(s_{1}\right) \ldots 1_{B_{k}}\left(s_{k}\right)$, where $A \in \mathscr{A}, B_{i} \in \mathscr{B}_{i}$ $(i=1, \ldots, k)$. For such $f$ we have

$$
\left(\mathbb{A}^{\mathbb{S}_{n}} f\right)\left(x ; s^{*}\right)=1_{\mathcal{A}}(x) \prod_{i=1}^{n} 1_{\mathcal{B}_{i}}\left(s_{i}\right) \prod_{j=n+1}^{k} \lambda\left(B_{j}\right) \quad \text { on } \quad X \times \mathcal{S}^{*}
$$

hence there exists a set $S^{* \prime} \in \mathscr{B}^{*}$ with $\lambda^{*}\left(S^{* \prime}\right)=1$ such that for each $s^{*}$ $=\left(s_{1}, s_{2}, \ldots\right) \in S^{* \prime}$

$$
\left(U \mathbb{E}^{\boldsymbol{s} n f}\right)_{\left(\mathrm{s}^{*}\right)}(x)=P_{s_{1}} 1_{\mathcal{A}}(x) \prod_{i=1}^{n} 1_{B_{i}}\left(s_{i+1}\right) \prod_{j=n+1}^{k} \lambda\left(B_{j}\right) \quad \text { on } \quad X .
$$

On the other hand, there exists a set $S^{* \prime \prime} \in \mathscr{B}^{*}$ with $\lambda^{*}\left(S^{* \prime \prime}\right)=1$ such that for each $s^{*}=\left(s_{1}, s_{2}, \ldots\right) \in \mathbb{S}^{* \prime \prime}$

Therefore

$$
(U f)_{\left(s^{*}\right)}(x)=P_{s_{1}} 1_{A}(x) \prod_{i=1}^{k} 1_{B_{i}}\left(s_{i+1}\right) \quad \text { on } \quad X .
$$

$$
\left(E^{\gamma++1} U f\right)_{\left(8^{*}\right)}(x)=P_{B_{1}} 1_{A}(x) \prod_{i=1}^{n} 1_{B_{i}}\left(s_{i+1}\right) \prod_{j=n+1}^{k} \lambda\left(B_{j}\right) \quad \text { on } \quad X
$$

which proves the lemma.

Wo say that a function $f$ defined on $X \times S^{*}$ does not depend essentially on parameters $s^{*}$ if $f$ is equivalent $\bmod \left(\mu \times \lambda^{*}\right)$ to some $\mathscr{C}_{0}$-measurable function.

For the sake of convonience, we will denote by $T$ the operator $E^{\varphi_{0}} \mathrm{U}$ throughout the remaining part of the paper.

1.5. LAтmar. If a function $f \in L_{1}\left(\mu \times \lambda^{*}\right)$ does not depend essentially on parametors $s^{*}$, then the funotions $f U$ and $f T$ atso do not depend essentially on parametors $s^{*}$ and $f U=f T$.

Proof. A function $f$ does not dopend essentially on parameters $s^{*}$, if $f E^{f_{0}}=f$. If $f D^{s_{0}}=f$, then $f D^{\phi_{1}}=f$ and, by Lemma 1.4, $f U D^{s_{0}}$ $=f Z^{\gamma_{1}} U=f U$. Moreover, if $f B^{\delta_{0}}=f$, then $f U=f B^{\delta_{0}} U=f T$, which completes the proof.

In the sequel the restriction of the operator $T$ to $L_{1}(\mu)$ will be denoted by the samo letter $T$. 
1.6. Remark. All the operators defined up to now and acting on $L_{1}(\mu)$ or $L_{1}\left(\mu \times \lambda^{*}\right)$ as well as their adjoints acting on $I_{\infty}(\mu)$ or $L_{\infty}\left(\mu \times \lambda^{*}\right)$, respectively, can be uniquely extended to the operators acting on arbitrary nonnegative functions defined on $X$ or $X \times S^{*}$, respectively (see e.g. [5], p. 4). These extensions will be denoted by the same lotters and, as it is easily seen, the Lemmas 1.3, 1.4 and 1.5 are still valid for them.

1.7. ExampLe. Let $\Phi=\left\{p_{s}: s \in S\right\}$ be a family of transformations $\varphi_{s}: X \rightarrow X$ and suppose that $\Phi$ is measurable, i.e. the mapping $(x, s) \rightarrow x \varphi_{s}$ is $\mathscr{A} \times \mathscr{B}$ - $\mathscr{A}$-measurable. Then the formula

$$
\left(x ; s^{*}\right) \varphi^{*}=\left(x \varphi_{s_{1}} ; s_{2}, s_{3} ; \ldots\right)
$$

defines a measurable transformation $\varphi^{*}$ on $\left(X \times S^{*}, \mathscr{Q}^{*} \times \mathscr{B}^{*}\right)$. Moreover, $\phi^{*}$ preserves the measure $\mu \times \lambda^{*}$ if and only if for every $A \in \Omega$

$$
\int_{S} \mu\left(A p_{s}^{-1}\right) d \lambda(s)=\mu(A) .
$$

If every transformation $\varphi_{s} \in \Phi$ is non-singular, then the family $\Phi$ determines a measurable family of sub-Markovian operators $\mathscr{P}=\left\{P_{8}: \quad s \in \mathbb{S}\right\}$ by the formula

$$
\left(P_{s} f\right)(x)=f\left(x \varphi_{s}\right) \quad \text { for } \quad f \in L_{\infty}(\mu) .
$$

The operator $U$ is now given by $(U f)\left(x ; s^{*}\right)=f\left(\left(x ; s^{*}\right) \varphi^{*}\right)$ and its iterates by

$$
\left(U^{n} f\right)\left(x ; s^{*}\right)=f\left(x \varphi_{s_{1}} \varphi_{s_{2}} \ldots \varphi_{s_{n}} ; s_{n+1}, \ldots\right) \text {. }
$$

2. The conservative part and invariant functions. Let $f_{0} \in L_{1}(\mu)$ be a function such that $\left\{f_{0}>0\right\}=X$ and let

$$
C_{T}=\left\{x \in X: \sum_{k=0}^{\infty}\left(f_{0} T^{k}\right)(x)=\infty\right\}
$$

The set $C_{r}$ does not depend on the choice of $f_{0} \in L_{1}(\mu)$ with the property $\left\{f_{0}>0\right\}=X$ and is called the conservative part of the sub-Markovian operator $T$ (see [5], Ch. II, 2.2). The set $D_{T^{\prime}}=X X O_{T^{\prime}}$ is called the dissipative part of $T$. The function $f_{0}$ considered as a function of the variables $\left(x ; s^{*}\right)$ has the following properties: $f_{0} \in L_{1}\left(\mu \times \lambda^{*}\right)$ and $\left\{f_{0}>0\right\}=X \times \mathcal{S}^{*}$. Therefore the set

$$
O_{U}=\left\{\left(x ; s^{*}\right) \in X \times S^{*}: \sum_{k=0}^{\infty}\left(f_{0} U^{l t}\right)\left(x ; s^{*}\right)=\infty\right\}
$$

is the conservative part of the sub-Markovian opexatox $U$. By Lemma 1.5 we have $f_{0} T^{n}=f_{0} U^{n}$ for $n=0,1,2, \ldots$ Hence

2.1. Theorear. $O_{U}=C_{x} \times \mathcal{S}^{*}$ on $X \times \mathcal{S}^{*}$.

A function $f\left(f \geqslant 0\right.$ or $\left.f \in L_{\infty}(\mu)\right)$ defined on $X$ is called harmonic on a set $C \in \mathscr{A}$ if $T f=f$ on $C$. We say that a function $f$ is $\mathscr{P}_{\text {-invariant on } O \text { if }}$ there exists a sot $S^{\prime} \in \mathscr{B}$ with $\lambda\left(S^{\prime}\right)=1$ such that for each $s \in S^{\prime} P_{s} f=f$ on o. Donote

and

$$
\mathscr{I}_{T},\left\{A \in \mathscr{A}: A \subseteq C_{T} \text { and } T 1_{\mathcal{A}}=1_{\mathcal{A}} \text { on } C_{T}\right\}
$$

$\mathscr{I}_{P}=\left\{A \in \mathscr{L}: A \subseteq O_{T}\right.$ and $1_{\mathcal{A}}$ is $\mathscr{P}$-invariant on $\left.C_{T}\right\}$.

2.2. Iismma. Wach function $f\left(f \geqslant 0\right.$ or $\left.f \in L_{\infty}(\mu)\right)$ defined on $X$ and harmonic on $C_{T}$ is $\mathscr{D}_{-i n v a r i a n t}$ on $C_{T}$. In particular, $\mathscr{I}_{T}=\mathscr{I}_{\mathscr{O}}$.

I'roof. By ([1.3], l'roposition V.5.2) if $0 \leqslant f<\infty$, then the equality Tf $f$ on $O_{T}$ is oquivalent to the $\mathscr{I}_{T}$-measurability of $f$ on $C_{T}$. Moreover, proceding as in tho proof of Theorem $\mathrm{A}$ in ([5], Ch. III), we observe that tho assumption of the finiteness of $f$ is not necessary here. Therefore it is sufficienti to prove the lemma for the functions of the form $f=1_{A}$, whoro $A \in \mathscr{A}$.

Let $T 1_{A}=1_{A}$ on $C_{T}$. By Theorem 2.1 this means that

$$
E^{\mathscr{C}_{0}} U 1_{A}=1_{A} \text { on } O_{U}
$$

Multiplying the above equality by the $\mathscr{C}_{0}$-measurable function $1_{A^{c}} 1 C_{U}$ $\left(A^{\circ}=X \backslash A\right)$ wo obtain

$$
D^{\sigma_{0}}\left(U 1_{A} \cdot 1_{A^{c}} \cdot 1 \sigma_{U}\right)=0 \text { on } X \times S^{*} \text {, }
$$

so $U 1_{A} \cdot 1_{A^{a}}=0$ on $\sigma_{U}$. Since $U 1_{A} \leqslant 1$ on $X \times S^{*}, U 1_{A} \leqslant 1_{A}$ on $C_{U}$. By ([13], Proposition V.5.2), $U 1_{\mathcal{A}}=1_{A}$ on $\sigma_{U}$, which proves the lemma. The following axample shows that in the above lemma $\sigma_{T}$ cannot be replaced by $X$, oven if the family $\mathscr{P}$ is determined by a family $\Phi$ of measure preserving transformations.

2.3. Examprex. Let $X$ bo a free group with two free generators $a$ and $b$ and lot $\mu$ be a counting measure on $(X, \mathscr{A})$, where $\mathscr{A}=2^{X}$. Let $S=\{a, b\}$ and $\lambda(\{a\})=\lambda(\{b\})=1 / 2$. For each $s \in S$ let $p_{s}: X \rightarrow X$ be the right translation of $X$. Lot $\mathscr{P}=\left\{P_{g}: s \in S\right\}$ be determined by the family of trunsformations $\Phi=\left\{\varphi_{s}: s \in \Phi\right\}$ as in Example 1.7 .

For wach $u \in[0,1)$ and $a$ fixed $d \in(0,1), d \neq 1 / 2$ put

$$
\begin{aligned}
& u w_{a}= \begin{cases}u /(2-2 d) & \text { for } \quad 0 \leqslant u<1-d, \\
(u+2 d-1) / 2 d & \text { for } \quad 1-d \leqslant u<1,\end{cases} \\
& u \psi_{b}\left\{\begin{array}{lll}
u / 2 d & \text { for } & 0 \leqslant u<d, \\
(u+1-2 d) /(2-2 d) & \text { for } & d \leqslant u<1 .
\end{array}\right.
\end{aligned}
$$

Since $\psi_{a}$ and $\psi_{b}$ are the invertible transformations on $[0,1)$, we can define $\psi_{a-1}=\psi_{a}^{-1}, \psi_{b-1}=\psi_{b}^{n-1}$.

Now, each element $x \in X$ can be written in the form $x=t_{1} t_{2} \ldots t_{n}$, whoro $t_{i} \in\left\{a, b, a^{-1}, b^{-1}\right\}$. Denote $A=[1 / 2,1)$ and put

$$
f(x)=f\left(t_{1} t_{2} \ldots t_{n}\right)=m\left(A \psi_{l_{1}}^{-1} \psi_{t_{2}}^{-1} \ldots \psi_{t_{n}}^{-1}\right)
$$


if $x=t_{1} t_{2} \ldots t_{n} \in X$. Here $m$ denotes the Lebesgue measure on $[0,1)$. The function $f$ does not depend on the representation of an element $x \in X$ and has the following properties:

$1^{\circ} 0 \leqslant f(x) \leqslant 1$ for each $x \in X$,

$2^{\circ} f$ is harmonic since for each $x=t_{1} t_{2} \ldots t_{n} \in X$ we have

$$
\begin{aligned}
\frac{1}{2} f\left(x \varphi_{a}\right)+\frac{1}{2} f\left(x \varphi_{b}\right) & =\frac{1}{2} m\left(A \psi_{t_{1}}^{-1} \ldots \psi_{t_{n}}^{-1} \psi_{a}^{-1}\right)+\frac{1}{2} m\left(A \psi_{t_{1}}^{-1} \ldots \psi_{l_{n}}^{-1} \psi_{b}^{-1}\right) \\
& =m\left(A \psi_{t_{1}}^{-1} \ldots \psi_{t_{n}}^{-1}\right)=f(x),
\end{aligned}
$$

$3^{\circ} f$ is not $\mathscr{P}$-invariant since for $\alpha=e$ (the identity in $X$ ) we have

and

$$
f(e)=f\left(a a^{-1}\right)=m\left(A \psi_{a}^{-1} \psi_{a}^{-1}-1\right)=m(A)=1 / 2
$$

$$
f\left(e p_{a}\right)=f(a)=m\left(A \psi_{a}^{-1}\right)=d \neq 1 / 2 .
$$

We say that a function $h\left(h \geqslant 0\right.$ or $\left.h \in L_{\infty}\left(\mu \times \lambda^{*}\right)\right)$ defined on $X \times 8^{*}$ is $U$-invariant on a set $C \in \mathscr{A} \times \mathscr{B}^{*}$ if $U h=h$ on $C$. Let

$$
\mathscr{I}_{U}=\left\{D \in \mathscr{A} \times \mathscr{B}^{*}: D \subseteq O_{U} \text { and } U 1_{D}=1_{D} \text { on } \sigma_{U}\right\} \text {. }
$$

2.4. THEOREM. Each function $h\left(h \geqslant 0\right.$ or $\left.h \in L_{\infty}\left(\mu \times \lambda^{*}\right)\right)$ defined on $X \times S^{*}$ and $U$-invariant on $O_{\sigma}$ does not depend essentially on parameters $s^{*}$ on $\mathscr{O}_{U}$. In particular, $\mathscr{I}_{U}=\mathscr{I}_{T} \times\left\{\varnothing, \mathbb{S}^{*}\right\} \bmod \left(\mu \times \lambda^{*}\right)$.

-Proof. Without loss of generality we may assume that the measure $\mu$ is finite (observe that the sets $C_{p}$ and $\sigma_{U}$ do not change if we take a measure $m$ equivalent to $\mu$ ). Moreover, it is sufficient to prove the theorem for the functions $h \in L_{\infty}\left(\mu \times \lambda^{*}\right)$.

Let a function $h \in L_{\infty}\left(\mu \times \lambda^{*}\right)$ satisfy $U h=h$ on $O_{U}$. Then, nsing ([5], Ch. $\Pi,(2.7))$, we obtain for every $m=1,2, \ldots$

$$
\sigma^{m} h=h \text { on } O_{U} \text {. }
$$

Using Lemma 1.4, (1), and Theorem 2.1. we have for $g=B^{\delta_{0}} h$ :

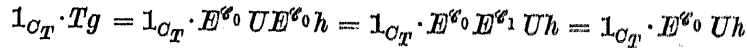

$$
\begin{aligned}
& =E^{\gamma_{0}}\left(1_{\sigma_{U}} \cdot U h\right)=W^{\delta_{0}}\left(1_{O_{U}} h\right)=1_{\sigma_{T}} g \text { on } \quad X \times S^{*},
\end{aligned}
$$

i.e. $T g=g$ on $C_{x}$. By Lemma $2.2 \mathrm{Ug}=g$ on $\sigma_{V}$, hence tor sach $m=1,2, \ldots$

$$
U^{m} g=g \text { on } O_{U} \text {. }
$$

On the other hand, using Lemma 1.4, (1), and Theorom 2.1 wo have for $m=0,1,2, \ldots$

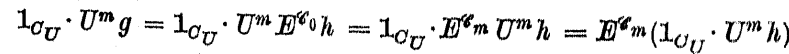

$$
\begin{aligned}
& =B^{\ell_{m}}\left(1_{\sigma_{U}} h\right)=1_{\sigma_{U}} \cdot E^{\ell_{m}} h \text { on } \quad X \times S^{*} \text {, }
\end{aligned}
$$

i.e. $U^{m} g=E^{6 m h}$ on $O_{U}$. By the martingale convergence theorem $\lim _{16} \mathbb{E}^{6} h=h$ on $X \times S^{*}$ (see [12], Oorollary $\amalg-2-12$ ). Therefore (3)

$$
\lim _{m \rightarrow \infty} U^{m} g=h \text { on } C_{U} .
$$

Comparing (2) and. (3) we have $h=g=E^{\ell_{0}} h$ on $C_{U}$.

In purticular, taking $h=1_{D}\left(D \in \mathscr{A} \times \mathscr{B}^{*}\right)$ we obtain the equality $\mathscr{I}_{U}=\mathscr{I}_{T} \times\left\{\emptyset, S^{*}\right\} \bmod \left(\mu \times \lambda^{*}\right)$, which completes the proof.

In the above theorem $C_{v}$ cannot be replaced by $X \times S^{*}$. This is the consequence of tixample 2.3 and of the following

2.5. Xroposirion. The following conditions are equivalent:

(i) each harmonic function $f \in L_{\infty}(\mu)$ is $\mathscr{P}$-invariant,

(ii) each $V$-invariant function $h \in L_{\infty}\left(\mu \times \lambda^{*}\right)$ does not depend essentially on parameters $s^{*}$.

Proof. Without loss of generality we may assume that the measure $\mu$ is finite.

To obtain the implication (i) $\Rightarrow$ (ii) one proceeds as in the proof of Theorem 2.4, replacing $C_{U}$ and $C_{T}$ by $X \times S^{*}$ and $X$, respectively, and using the assumption (i) instead of Lemma 2.2 .

For the proof of the converse implication assume that $T f=f$ for a $f \in L_{\infty}(\mu)$. Using Lemma 1.4 we obtain for $m=0,1,2, \ldots$

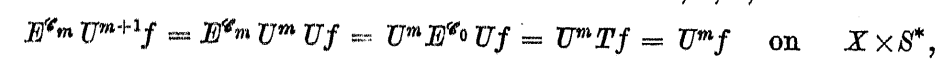

which means that the sequence $U^{m} f$ forms a martingale with respect to the increasing sequence of sub- $\sigma$-fields $\mathscr{C}_{m}$. Moreover, the functions $U^{m} f$ are uniformly bounded in $L_{\infty}\left(\mu \times \lambda^{*}\right)$, and since the measure $\mu$ is finite, also in $L_{1}\left(\mu \times \lambda^{*}\right)$. By the martingale convergence theorem there exists. a function $h \in I_{\infty}\left(\mu \times \lambda^{*}\right)$ such that

$$
\lim _{m \rightarrow \infty} U^{m} f=h \quad \text { on } \quad X \times S^{*}
$$

(see [12], Theorem IV-1-2). Moreover, the convergence in (4) holds in the norm topology of $I_{1}\left(\mu \times \lambda^{*}\right)$ and in the weak*-topology of $L_{\infty}\left(\mu \times \lambda^{*}\right)$. This implies that the martingalo $U^{m} f$ is regular (see [12], Proposition IV-2-3), henee

$$
U^{m} f=D^{6} m h \quad \text { for } \quad m=0,1,2, \ldots
$$

and, by the weak ${ }^{*}$-continuity of the operator $U$, that $U h=h$. The assumption (ii) implios that $h=\mathbb{B}^{\mathbb{S}_{0}} h$ and from (5) applied to $m=0$ we obtain $f=\mathbb{E}^{x_{0}} h=h$. Therefore the function $f$ is $\mathscr{P}$-invariant, which completes. the proof.

Now, we turn to the investigation of functions $h$ defined on $X \times S^{*}$ and satisfying the equation $h U=h$.

The following result generalizes the first part of Satz 4 in [11]. 
2.6. THEOREM. Each function $h \in I_{1}\left(\mu \times \lambda^{*}\right)$ satisfying the equation $h U=h$ does not depend essentially on parameters $8^{*}$.

Proof. If $h \in L_{1}\left(\mu \times \lambda^{*}\right)$ and $h U=h$, then, using Lemma 1.4, we have

$$
h E^{\varepsilon_{0}}-h=h U^{m} D^{\delta_{0}}-h U^{m}=\left(h D^{\mathscr{C}_{m}}-h\right) U^{m} .
$$

Since for each $m=1,2, \ldots U^{m}$ is a contraction on $L_{1}\left(\mu \times \lambda^{*}\right)$, Iheneo

$$
\left\|h \mathbb{E}^{r_{0}}-h\right\|_{L_{1}\left(\mu \times \lambda^{*}\right)} \leqslant\left\|h H^{\gamma_{m}}-h\right\|_{x_{1}\left(\mu \times \lambda^{*}\right)} \text {. }
$$

By the $L_{1}(\mu)$-valued martingale convergence theorem, $\lim _{m \rightarrow \infty} h H^{6} m=h$ in the norm of $L_{1}\left(\mu \times \lambda^{*}\right)$ (see [3], Theorem 2.1 (a)). Therefore $\left.h t\right)^{\gamma^{4}}=h$, which completes the proof.

2.7. Remark. For each finite signed measure $\varrho$ defined on $\mathscr{A} \times \mathscr{B}^{*}$ and absolutely continuous with respect to $\mu \times \lambda^{*}\left(\varrho \ll \mu \times \lambda^{*}\right)$ the formula

$$
(\varrho U)(D)=\iint_{X \times S^{*}} U 1_{D} d \varrho \quad\left(D \in \mathscr{A} \times \mathscr{B}^{*}\right)
$$

defines a finite signed measure $\varrho U \ll \mu \times \lambda^{*}$. Moreover, if $h \in L_{1}\left(\mu \times \lambda^{*}\right)$ and $\varrho(D)=\iint_{D} h d\left(\mu \times \lambda^{*}\right)$, then $h U=d(\varrho U) / \dot{d}\left(\mu \times \lambda^{*}\right)$ on $X \times S^{*}$, where $d(\varrho U) / d\left(\mu \times \lambda^{*}\right)$ denotes the Radon-Nikodym derivativo.

The action of the operator $T$ on a mensure $\nu$ definod on $. \alpha, v \ll \mu$, is defined similarly.

Theorem 2.6 formulated in the language of measures says that oach finite signed measure $\varrho$ defined on $\mathscr{A} \times \mathscr{B}^{*}, \varrho \leqslant \mu \times \lambda^{*}$ and invariant for the operator $U$ (i.e. satisfying the equation $\varrho U=\varrho$ ) is of the form $\varrho$ $=\nu \times \lambda^{*}$ for a finite signed measure $\nu$ on $\mathscr{A}$. Moreover, wo have $\nu \ll \mu$ and $\nu T=\nu$, which follows from Lemma 1.5.

2.8. THEOREM. Suppose that $\mu$ is $\sigma$-finite and subinvariant for the operator $T$ (i.e. $1 T \leqslant 1$ on $X$ ). Then each funotion $h \in L_{p}\left(\mu \times \lambda^{*}\right)$ with 1 $\leqslant p<\infty$ satisfying the equation $h U=h$ does not depend essentially on
parameters $s^{*}$.

Proof. If $1 T \leqslant 1$ on $X$, then $1 U \leqslant 1$ on $X \times S^{*}$ by Lemma 1.5 . Therefore the operator $U$, which is a contraction on $L_{1}\left(\mu \times \lambda^{*}\right)$, is simultianeously a contraction on $L_{\infty}\left(\mu \times \lambda^{*}\right)$. By the interpolation theorem (see) [4], Theorem VI.10.11) $U$ is a contraction on anch $L_{p}\left(\mu \times \lambda^{*}\right)$ with $1 \leqslant p$
$\leqslant \infty$.

Now, let $h U=h$ for a $h \in I_{p}\left(\mu \times \lambda^{*}\right), 1 \leqslant p<\infty$. Analogously as in the proof of Theorem 2.6 we obtain

$$
\left\|h E^{\ell_{0}}-h\right\|_{x_{p p}\left(\mu \times \lambda^{*}\right)} \leqslant\left\|h E^{\delta_{m}}-h\right\|_{L_{L_{p}\left(\mu \times \lambda^{*}\right)}} .
$$

By the $L_{p}(\mu)$-valued martingale convergenco theorem, $\lim _{m \rightarrow \infty} h D^{S_{m}}=-=h$ in the norm of $I_{p}\left(\mu \times \lambda^{*}\right)$ (see [3], Theorem 2.1 (a)). Therefore $h \mathbb{B}^{\mathscr{S}_{0}}=h$, which completes the proof.

2.9. Remark. Theorem 2.6 is not true if $h$ is not integrable (and only positive) and Theorem 2.8 is false for $p=\infty$. To see this let us consider the family $\mathscr{P}=\left\{P_{a}, P_{b}\right\}$ defined in Example 2.3. As we have seen, there exists a function $h_{0} \in L_{\infty}^{+}\left(\mu \times \lambda^{*}\right)$ such that $U h_{0}=h_{0}$ and $h_{0}$ depends essentially on paramoters $s^{*}$. Since the measure $\mu \times \lambda^{*}$ in Example 2.3 is $\varphi^{*}$. invariant, it follows that the measure $d \varrho=h_{0} d\left(\mu \times \lambda^{*}\right)$ is also $p^{*}$-invariant and so $h_{0} U=h_{0}$.

3. Exgodic theorems. In gencral the restriction of the $\sigma$-finite measure $\mu$ to the $\sigma$-ficld $\mathscr{S}_{T}$ of subsets of $O_{x}$ is not $\sigma$-finite. Howerer

3.1. Lrimms (see [5], Oh. III, (3.7)). The conservative part of the operator $T$ decomposes uniquely $\bmod \mu$ into a disjoint union

$$
O_{T}=C_{x}^{\mathrm{1}} \cup O_{x}^{2}
$$

where $O_{T^{\prime}}^{1} \in \mathscr{I}_{T}, O_{T}^{1}=\bigcup_{n=1}^{\infty} A_{n}$ for an increasing sequence of sets $A_{n} \in \mathscr{I}_{T}$ with $\mu\left(A_{n}\right)<\infty$, and ${ }^{n-1}(A)=0$ or $\infty$ for every set $A \in \mathscr{I}_{T}, A \subseteq C_{T}^{2}$. Let $\mathscr{I}_{T}^{1}$ be the restriotion of the $\sigma$-field $\mathscr{I}_{T}$ to $O_{T}^{1}$. Then $\left(O_{T^{1}}^{1}, \mathscr{J}_{T^{1}}^{1}, \mu\right)$ is a $\sigma$-finite measure space.

Given a function $f \in L_{1}(\mu)$ supported on $O_{T}^{1}, A^{\sigma^{1}} f$ will denote the unique $\mathscr{I}_{T^{1}}^{1}$-measurable function defined on $C_{T}^{1}$ and satisfying for every $A \in \mathscr{I}_{T^{1}}^{1}$ the equation $\int_{\mathcal{A}} f d \mu=\int_{\mathcal{L}} E^{\mathcal{G}_{T}^{1}} f d \mu$.

Analogously, we have

3.2. Lemana. The conservative part of the operator $U$ decomposes uniquely $\bmod \left(\mu \times \lambda^{*}\right)$ into a disjoint union

$$
O_{U}=O_{U}^{\mathrm{1}} \cup O_{U}^{2}
$$

where $O_{U}^{1} \in \mathscr{I}_{U}, O_{U}^{1}=\bigcup_{n=1}^{\infty} D_{n}$ for an increasing sequence of sets $D_{n} \in \mathscr{I}_{U}$ with $\left(\mu \times \lambda^{*}\right)\left(D_{n}\right)<\infty$, and $d^{n=1}\left(\mu \times \lambda^{*}\right)(D)=0$ or $\infty$ for every set $D \in \mathscr{I}_{U}, D \subseteq O_{U}^{2}$. Let $\mathscr{I}_{I J}^{1}$ be the restriction, of the $\sigma=$ field $\mathscr{I}_{V}$ to $C_{V}^{1}$. Then $\left(C_{U T}^{1}, \mathscr{I}_{U}^{1}, \mu \times \lambda^{*}\right)$ is a orfinite measure space.

Givon. a function $h \in L_{1}\left(\mu \times \lambda^{*}\right)$ supported on $O_{V}^{1}, E^{s / 1} t h$ denotes the unique $\mathscr{I}_{U}^{1}$-mousurable function defined on $O_{U}^{1}$ and satisfying for every $D \in \mathscr{I}_{V}^{1}$ the equation.

$$
\iint_{D} h d\left(\mu \times \lambda^{*}\right)=\iint_{D} E^{\mathscr{\sigma}^{1}} U h d\left(\mu \times \lambda^{*}\right) .
$$

7 - Studia Math. 74.2 
3.3. THEOREM. (1) $O_{U}^{1}=O_{T}^{1} \times S^{*}$ on $X \times S^{*}$,

(2) $C_{U}^{2}=C_{T}^{2} \times S^{*}$ on $X \times S^{*}$,

(3) $\mathscr{I}_{U}^{1}=\mathscr{I}_{T}^{1} \times\left\{\varnothing, S^{*}\right\} \bmod \left(\mu \times \lambda^{*}\right)$.

Proof. Let $A_{n}$ be the sets appearing in Lemma 3.1. Wo have $A_{n} \times$ $\times S^{*} \in \mathscr{I}_{T} \times\left\{\varnothing, S^{*}\right\}$ and $\mathscr{I}_{T} \times\left\{\varnothing, S^{*}\right\}=\mathscr{I}_{\sigma} \bmod \left(\mu \times \lambda^{*}\right)$, by Theorem 2.4. Moreover, $\left(\mu \times \lambda^{*}\right)\left(A_{n} \times \mathcal{S}^{*}\right)=\mu\left(A_{n}\right)<\infty$. Therefore

$$
O_{T}^{1} \times S^{*}=\bigcup_{n=1}^{\infty} A_{n} \times S^{*} \subseteq O_{U}^{1} \quad \text { on } \quad X \times S^{*} .
$$

On the other hand, if $D_{n}$ are the sets appearing in Lomma 3.2, then $D_{n} \in \mathscr{I}_{U T}=\mathscr{I}_{T} \times\left\{\varnothing, s^{*}\right\} \bmod \left(\mu \times \lambda^{*}\right)$, by Theorem 2.4. Therefore $D_{n}$ $=B_{n} \times S^{*}$ on $X \times S^{*}$ for an increasing sequence of setis $B_{n} \in \mathscr{I}_{T^{*}}$. Moreover, $\mu\left(B_{n}\right)=\left(\mu \times \lambda^{*}\right)\left(D_{n}\right)<\infty$. Hence

$$
C_{U}^{1}=\bigcup_{n=1}^{\infty} D_{n}=\bigcup_{n=1}^{\infty} B_{n} \times S^{*} \subseteq O_{T}^{1} \times S^{*} \quad \text { on } \quad X \times S^{*},
$$

which proves (1). Furthermore, (2) follows from (1) and from Theorem 2.1, and (3) follows from (1) and from Theorem 2.4, which completes the proof.

The following theorem generalizes the random ergodic theorem of Ryll-Nardzewski ([15], Theorem 1) and of Nawrotzki ([111], Satz 4). In the formulation of this theorem the symbol $P_{s_{k}}$ denotos the extension of the operator $P_{s_{k}} \in \mathscr{P}$ to the class of all nonnegative functions defined on $X$ (see Remark 1.6).

3.4. THEOREM. Suppose that $\mu$ is offinite and subinvariant for the operator T. Then for each function $f\left(x ; s^{*}\right) \in X_{p}^{+}\left(\mu \times \lambda^{*}\right)$ with $1 \leqslant p<\infty$ there exist a function $f^{*}(x) \in I_{p}^{+}(\mu)$ and a set $S^{* \prime} \in \mathscr{B}^{*}$ with $\lambda^{*}\left(S^{* \prime}\right)=1$ such that for each $s^{*}=\left(s_{1}, s_{2}, \ldots\right) \in S^{* \prime}$

(4) $\quad \lim _{n \rightarrow \infty} \frac{1}{n} \sum_{k=0}^{n-1} P_{s_{1}} P_{s_{2}} \ldots P_{s_{l}} f_{\left(s_{l}+1, \ldots\right)}(x)=f^{*}(x) \quad$ on $\quad X$, and if $p>1$,

(5) $\quad \lim _{n \rightarrow \infty}\left(\int_{\bar{X}}\left|\frac{1}{n} \sum_{k=0}^{n-1} P_{s_{1}} P_{s_{2}} \ldots P_{s_{k}} f_{\left(s_{k+1} \ldots \ldots\right)}(x)-f^{*}(x)\right|^{p} d \mu(x)\right)^{1 / p}=0$.

Moreover, the limit function satisfies

$$
f^{*}=\left\{\begin{array}{lll}
0 & \text { on } & \left(D_{T} \cup O_{T}^{2}\right) \times S^{*}, \\
E^{\mathcal{G}_{T}^{1} \times\left\{\emptyset, S^{*}\right\}}\left(1_{O_{T}^{1}}^{1} f\right) & \text { on } & O_{T}^{1} \times S^{*} .
\end{array}\right.
$$

Proof. From the assumption that $1 T \leqslant 1$ on $\mathrm{X}$ and from Lomma 1.5 it follows that $1 U \leqslant 1$ on $X \times S^{*}$. By the pointwise ergodic theorem applied to the operator $U$ it follows that for each function $f \in I_{p}^{+-}\left(\mu \times \lambda^{*}\right)$ there exists a function $f^{*} \in T_{j}^{*}\left(\mu \times \lambda^{*}\right)$ such that

$$
\lim _{n \rightarrow \infty} \frac{1}{n} \sum_{k=0}^{n-1} U^{k} f=f^{*} \quad \text { on } \quad X \times S^{*}
$$

ind

$$
f^{*}=\left\{\begin{array}{lll}
0 & \text { on } & D_{U} \cup O_{U}^{2}, \\
W^{F^{*}}\left(1_{C_{U T}^{1}}^{1} f\right) & \text { on } & O_{U}^{1}
\end{array}\right.
$$

(for $f \in L_{1}^{+}\left(\mu \times \lambda^{*}\right)-$-Hes [5], Oh. VIr, Theorem B; for $f \in L_{p}^{+}\left(\mu \times \lambda^{*}\right)$ (7) holds by [4], Theorem VIrr.6.6 and (8) follows from the just mentioned theorems).

Using Theorem 2.1, Lomma 3.3 and (8) we conclude that the limit function $f^{*}$ does not depend essentially on parameters $s^{*}$ and has the

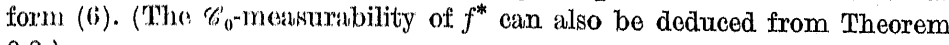
2.8.)

By the Fubini theorem, Lemma 1.3 and (7) it follows that (4) holds for $\lambda^{*}$-almost all $s^{*}$. Finally, (5) follows from (4), the Fubini theorem and the fact that for $f \in I_{j}^{\dagger}\left(\mu \times \lambda^{*}\right)$ with $1<p<\infty$ we have

$$
\sup _{n=1,2, \ldots} 1 . \sum_{n=0}^{n-1} U_{k=0}^{k} f \in L_{p}^{+}\left(\mu \times \lambda^{*}\right)
$$

(see [4], Theorm VIIT.6.8). The proof of Theorem 3.4 his hereby been completied.

The following result is analogous to Theorem 2.8.

3.5. OoRoLriAXY. Suppose that $\mu$ is o-finite and subinvariant for the operator $T$ and $1 \leqslant p<\infty$. Then

(i) each U-invariant function $h \in L_{p}^{+}\left(\mu \times \lambda^{*}\right)$ does not depend essentially on parameters $s^{*}$ and

(ii) each harmonic funstion $f \in T_{p}^{+}(\mu)$ is $\mathscr{P}$-invariant.

Proof. (i) follows from Theorem 3.4. Now, if $f \in X_{p}^{+}(\mu)$ for $1 \leqslant p<\infty$ and $T f=f$, then

$$
f=\left\{\begin{array}{lll}
0 & \text { on } & D_{T^{\prime}} \cup O_{T}^{2}, \\
W^{*} l^{\prime}\left(\mathrm{1}_{\sigma_{T}}^{1} f\right) & \text { on } & O_{T}^{1}
\end{array}\right.
$$

(see [5], (Th. VII, Theorem .B). Therefore the 99 -invariance of $f$ follows from the formula (6) in Theorem 3.4 and from the fact that the limit function $f^{*}$ in Theorem 3.4 is 98 -invariant.

The part (i) of the above corollary can also be deduced directly from Theorom 2.8 .

Observe that by Lemma 1.2, Lemma 1.5 and by the definitions of 
the operators $U$ and $T$ it follows that

$$
\int_{X} f T \cdot g d \mu=\int_{S} \int_{X} f P_{s} \cdot g d \mu d \lambda(s)
$$

for every $f \in L_{1}(\mu), g \in L_{\infty}(\mu)$. Henee, if $A \in \mathscr{A}$, then

$$
\mu T(A)=\int_{X} 1 T \cdot 1_{A} d \mu=\int_{S} \int_{X} 1 P_{s} \cdot 1_{d} d \mu d \lambda(s)=\int_{S} \mu P_{B}(\Lambda) d \lambda(s) .
$$

In particular, the measure $\mu$ is invariant for the opentior ' $L$ ' if for overy $A \in \mathscr{A}$ we have

$$
\int_{S} \mu P_{s}(A) d \lambda(s)=\mu(A),
$$

Let us introduce a stronger condition. Namely, we say that $\mu$ is $\mathscr{P}$-invariant if for every $A \in \mathscr{A}$ we have

$$
\mu P_{s}(A)=\mu(A) \lambda \text {-almost overywhere. }
$$

Now, we shall formulate a particular case of Theorem 3.4, whon the measure $\mu$ is finite and $T$-invariant and $p=1$.

3.6. THEOREM. Suppose that $\mu$ is finite and invariant for tho operator $T$. Then for each function $f\left(x ; s^{*}\right) \in I_{1}^{+-}\left(\mu \times \lambda^{*}\right)$ there exist a funotion $f^{*}(x) \in L_{1}^{+}(\mu)$ and a set $S^{* \prime} \in \mathscr{B}^{*}$ with $\lambda^{*}\left(S^{* \prime}\right)=1$ suoh that for enory $s^{*},\left(s_{1}, s_{2}, \ldots\right) \in S^{* \prime}$

$$
\lim _{n \rightarrow \infty} \frac{1}{n} \sum_{k=0}^{n-1} P_{s_{1}} P_{s_{2}} \ldots P_{s_{k}} f_{\left(s_{k+1}, \ldots\right)}(x)=f^{*}(x) \quad \text { on } \quad X
$$

and if

$$
\int_{X \times S^{*}} f \log ^{+} f d\left(\mu \times \lambda^{*}\right)<\infty
$$

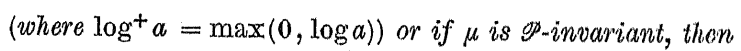

$$
\text { (11) } \quad \lim _{n \rightarrow \infty} \int_{X}\left|\frac{1}{n} \sum_{k=0}^{n-1} P_{s_{1}} P_{s_{2}} \ldots P_{s_{k}} f_{\left(s_{k+1}+\ldots\right)}(x) \ldots f^{*}(x)\right| d \mu(x) \ldots 0 \text {. }
$$

Moreover, the limit funotion satisfies

$$
f^{*}=E^{\mathscr{I}} T^{\times\left\{\varnothing, S^{*}\right\}} f \quad \text { om } \quad X \times S^{*} .
$$

Proof. The convergence of the ergodic averuges (9) was proved in Theorem 3.4. Since $\mu$ is finite and invariant for $T$, so $O_{\eta^{1}}^{1}, Q_{T^{4}}, X$ and formula (12) follows from (6). If $f$ satistios (10), then

$$
\sup _{n=1,2, \ldots} 1 \sum_{k=0}^{n-1} U^{k} f \in I_{1}^{++}\left(\mu \times \lambda^{*}\right)
$$

(seo [4], Theorom VIrr.6.8) which, analogously as in the proof of (5) in Theorom 3.4, gives (11.).

Now, suppose that $\mu$ is $\mathscr{P}$-invariant. Then, as it is easily seen, we have $W^{\mathscr{E}} U f:=U W^{\delta^{8}} f$ for aw nonnegutive function $f$, where $\mathscr{E}=\{\varnothing, X\} \times \mathscr{B}^{*}$. Thesrefors, for moh $f \in L_{1}^{+}\left(\mu \times \lambda^{*}\right)$

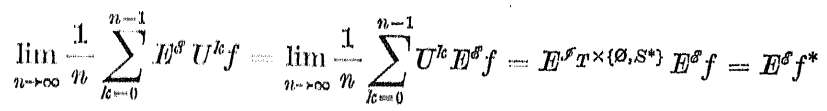

on $X \times S^{*}$. Oonserpurantily, there existis a set $S^{* \prime \prime} \in \mathscr{B}^{*}$ with $\lambda^{*}\left(S^{* \prime \prime}\right)=1$ such thati for mols $s^{*} \quad\left(s_{1}, s_{2}, \ldots\right) \in \mathrm{S}^{* * \prime \prime}$

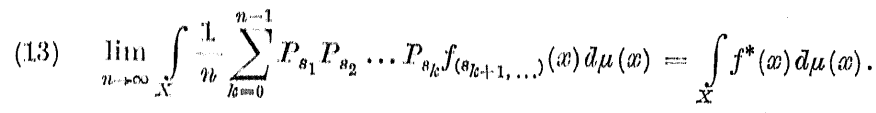

The formulas (9) and (13) imply (11), which complotes the proof.

3.7. Romark. The additional assumption (10) in the above theorem is essential oven if $f$ doos not depend essentially on parameters $s^{*}$. A suitable examplo will bo givon in Section 4.

Applying Theorom 3.6 and the Banach-Steinhaus theorem we obtain

3.8. Oorolidary. Suppose that $\mu$ is finite and $\mathscr{P}$-invariant and let the offield $\&$ bo countably generated. Then there exists a set $\mathcal{S}^{* \prime} \in \mathscr{B}^{*}$ with $\lambda^{*}\left(S^{* \prime}\right)=$ I. such that for every $s^{*}=\left(s_{1}, s_{2}, \ldots\right) \in S^{* \prime}$ and for every $f \in L_{1}(\mu)$ we have

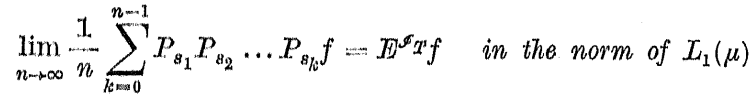

and

$$
\lim _{n \rightarrow \infty} \frac{1}{n} \sum_{k=0}^{n-1} f P_{s_{1}} P_{s_{2}} \ldots P_{s_{k}}=E^{\curvearrowleft} T f
$$

in the weak topology of $L_{1}(\mu)$.

The following axamplo shows that tho above corollary is falso if the

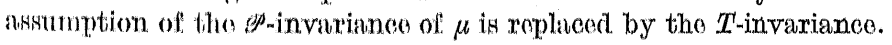

3.9. Wxampln. Leti $(X, \infty, \mu)$ to the interval $[0, x)$ with the Borel

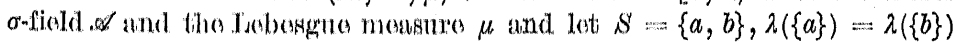
$\therefore 1 / 2$. Lat, $\varphi_{a}$ and $\varphi_{b}$ denote tho transiformations on $X$ given by $x \varphi_{a}=x / 2$ and $x p_{b}, \infty / 2+1 / 2$ for $x \in X$ and lot $\mathscr{P}=\left\{P_{a}, P_{b}\right\}$ bo determined by the fiumily $\Phi=\left\{\varphi_{a}, \psi_{l}\right\}$ as in Example 1.7. Obviously, the measure $\mu$ is $\boldsymbol{T}$ invariant. Moreover, as it is easily seen, for each $s^{*}=\left(s_{1}, s_{2}, \ldots\right) \in \mathbb{S}^{*}$ we have

$$
\lim _{n \rightarrow \infty} 1 P_{B_{1}} P_{B_{2}} \ldots P_{B_{n}}(\infty)=0 \text { on } \quad X
$$


which implies that in (14) the convergenee does not hold erven in the woak topology of $L_{1}(\mu)$ and that (15) is false aven for a fixed function $f \equiv 1$.

Using the random ergodic theorem we oun give another proof of the following well-known result (seo [10], Oh. VIIT, Theorem 7 ind [14], Ch. 5, Lemma 1).

3.10. OonoLxarx. Suppose that $\mu$ is finite and invariant for the operator T. If $\mathscr{P}$ is a commutative farnity of operators, i.e. if

$$
P_{s} P_{t}=P_{t} P_{s} \text { for all } 8, l \in \mathrm{S} \text {, }
$$

then $\mu$ is P्P-invariant.

Proof. Fix a set $A \in \mathscr{A}$. Tho assumption (1, i) implines that for avery $s^{*}=\left(s_{1}, s_{2}, \ldots\right) \in S^{*}$ and every $n: 0,1,2, \ldots$ wo have

$$
\left\langle 1 P_{s_{1}} P_{s_{2}} \ldots P_{s_{n}}, 1_{A}\right\rangle=\left\langle 1 P_{s_{n}} P_{s_{n-1}} \ldots P_{n_{1}}^{\prime}, I_{A}\right\rangle,
$$

where $\langle f, g\rangle=\int_{x} f g d \mu$ for $f \in I_{1}(\mu), g \in I_{\infty}(\mu)$. Sinco $1 T \ldots \ldots$, Theorom 3.6 implies that there exists at set $S^{* \prime} \in \mathscr{B}^{*}$ with $\lambda^{*}\left(\mathcal{S}^{* \prime}\right)=1$ such that

$$
\lim _{n \rightarrow \infty} \frac{1}{n} \sum_{k_{k=0}}^{n-1}\left\langle 1 P_{s_{1}} P_{s_{2}} \ldots P_{s_{k}}, 1_{A}\right\rangle=\left\langle 1,1_{A}\right\rangle
$$

for every $s^{*}=\left(s_{1}, s_{2}, \ldots\right) \in S^{* * \prime}$.

On the other hand, the equation $1 T: \ldots$ moans that the soguonce $\left\langle 1 P_{s_{n}} P_{s_{n-1}} \ldots P_{s_{1}}, 1_{A}\right\rangle$ forms a martingalo with rosposet to the incroasing sequence of $\sigma$-fields $\mathscr{B}_{1} \times \mathscr{B}_{2} \times \ldots \times \mathscr{B}_{n} \times\left\{\varnothing, \mathbb{S}_{n+1.1}\right\} \times\left\{\varnothing, \mathbb{S}_{n+2}\right\} \times \ldots$ Moroover, this martingale is uniformly bounded in $I_{\infty}\left(\lambda^{*}\right)$. By the martingals convergence theorem there exists a function $h_{A} \in I_{1}^{*}\left(\lambda^{*}\right)$ and a net $S^{* \prime \prime \prime} \in O B^{*}$ with $\lambda^{*}\left(S^{* \prime \prime}\right)=1$ stch that for every $s^{*}:=\left(s_{1}, s_{2}, \ldots\right) \in S^{* \prime \prime}$

and

$$
\lim _{n \rightarrow \infty}\left\langle 1 P_{s_{n}} P_{s_{n-1}} \ldots P_{s_{1}}, 1_{A}\right\rangle=h_{\perp L}\left(s_{1}, s_{2}, \ldots\right)
$$

$$
\left\langle 1 P_{s_{n}} P_{s_{n-1}} \ldots P_{s_{1}}, 1_{A}\right\rangle \ldots U^{r} n h_{A}\left(s_{1}, s_{2}, \ldots\right)
$$

for $n=0,1,2, \ldots$ (seo [12], Theorem IV-1-2 and Proporition IV-2-3).

Using (17), (18) and (19) we olbtain $h_{\mathcal{A}}$ const $\left\langle 1,1_{A}\right\rangle \lambda^{*}-\{1,0$. By $(20)\left\langle 1 P_{s_{1}}, 1_{d}\right\rangle=\left\langle 1,1_{d}\right\rangle$ tor $\lambda_{1}$-almost all $s_{1} \in \mathbb{S}_{1}$, which completos the proof.

4. Example. In this section wo shall constructi a probubility spaces $(X, \mathscr{A}, \mu)$, a measurable family $\mathscr{P}=\left\{P_{s}: s \in S^{S}\right\}$ of sul-Minkovian oparators on $L_{\infty}(\mu)$ with $\mu T=\mu$ and a function $f \in L_{1}^{+}(\mu)$ such that

$$
\limsup _{n \rightarrow \infty} E^{\delta}\left(\frac{1}{n} U^{n} f\right)=+\infty \mu \times \lambda^{*} \text {-a.o., }
$$

where $\mathscr{E}=\{\varnothing, X\} \times \mathscr{B}^{*}$. This gives

$$
\limsup _{n \rightarrow \infty} Z^{8}\left(\frac{1}{n} \sum_{l=0}^{n-1} U^{k_{0}} f\right)=+\infty \mu \times \lambda^{*} \text {-a.e., }
$$

and shows that tho adilitional assumption (10) in Theorem 3.6 is essential. Jut $(S, C B, \lambda)$ bo a nontrivial probability space and let

$$
X=\prod_{i=1}^{1} S_{i}, \quad \mathscr{W}=\prod_{i=\infty}^{0} \mathscr{B}_{i}, \quad \mu=\prod_{i=-\infty}^{0} \lambda_{i},
$$

whoro $s_{i} s, A_{i} \ldots, \lambda_{i}=\lambda$ lor $i=0,-1,-2, \ldots$ Lot $\varphi_{s}$ bo a transformatition on $X$ given by

$$
\left(\ldots, s_{-2}, s_{\cdots 1}, s_{0}\right) \psi_{s}:\left(\ldots, s_{-1}, s_{0}, s\right),
$$

und lot $\mathscr{P}^{\prime},\left\{I_{A}^{\prime}: \Delta \in S\right\}$ bo dotermined by the family of transformations $\Phi=\left\{p_{s}: s \in S\right\}$ as in Examplo 1.7. (Noto that if $\mathscr{B}$ contains all the sets of the form $\{s\}$, whoro $s \in S$ and if $\lambda(\{s\})>0$ for every $s \in S$, then each $p_{s} \in \Phi$ is a nonsingular transformation on $(X, \mathscr{A}, \mu)$.) Observe that $\varphi^{*}: X \times$ $\times S^{*} \rightarrow X \times S^{*}$ is simply a two-sided Bernoulli shift and $\mathscr{E}$ consists of those evontis which dopend only on the futuro. Since $p^{*}$ preserves the measure $\mu \times \lambda^{*}$, wo have $\mu T=\mu$.

Wo shall constiruct a function $f \in L_{1}^{+*}\left(X \times S^{*}, \mathscr{A} \times \mathscr{B}^{*}, \mu \times \lambda^{*}\right)$ such that $f$ is $\mathscr{A} \times\left\{\varnothing, s^{*}\right\}$-mousuruble and

$$
\left(\mu \times \lambda^{*}\right)\left(\left\{\mathbb{W}^{s^{n}} U^{n} f \geqslant n \log n \text { i.o. }\right\}\right)=1 .
$$

Obviously such a function $f$ satisfies (1).

Ohoose $\because B \in \mathscr{B}$ with $0<\lambda(B)<1$ and denote $p=\lambda(B), q=1-p$. Let $\left\{a_{n}\right\}_{n=0}^{\infty}$ bo a strictly increasing sequence of positive real numbers. Assume that thero exists a strictly increasing sequence of nonnegative integers $\left\{b_{n}\right\}_{n=0}^{\infty}$ with $b_{0}=0$ such that

$$
\begin{gathered}
\sum_{n \rightarrow 1}^{\infty}\left(a_{n_{n}-1}-p a_{b_{n+1}-1}\right)^{-} p^{n}<\infty, \\
\lim _{n \rightarrow \infty} a_{n_{n}-1} p^{n}=0 \\
\sum_{n \rightarrow \infty}^{\infty} b_{n} p^{n}=\infty \\
\liminf _{n \rightarrow \infty} \frac{b_{n+1}}{b_{n}}>1
\end{gathered}
$$

whore $(x)^{-}$donotes the negative part of $x$. Wo shall prove that under these assumptions thero exists an $f \in L_{1}\left(\mu \times \lambda^{*}\right)$ such that

$$
\left(\mu \times \lambda^{*}\right)\left(\left\{H^{0^{8}} U^{n} f \geqslant a_{n l} \text { i.o. }\right\}\right)=1 \text {. }
$$


Let $A_{0}=X \times S^{*}, A_{n}=\bigcap_{i=1}^{n} B_{b_{i}-1}(n=1,2, \ldots)$ und $\quad \sigma_{n} \quad A_{n-1} \backslash A_{n}$ where $B_{n}=\ldots \times S_{-n-2} \times S_{-n-1} \times B \times S_{\ldots n+1} \times S_{\cdots n+2} \times \ldots \subseteq X \times S^{\psi *}(n=0$, $1, \ldots)$. Denote $a_{n}=a_{b_{n+1}-1}(n=0,1,2, \ldots)$ and $\beta_{n} \quad q^{-1}\left(a_{n-1}-p \alpha_{n}\right)$ $(n=1,2, \ldots)$. Let

$$
f=\sum_{n=1}^{\infty} \beta_{n} 1_{t t_{n}}
$$

Since $\sum_{n=1}^{N} \beta_{n} p^{n-1} q=\alpha_{0}-\alpha_{N} p^{N}$, wo infor from (3) and (1) that $f$ is integrntulds and $\int_{X \times S^{*}} \int_{n=1} f d\left(\mu \times \lambda^{*}\right)=a_{b_{1}-1}$. Denoto

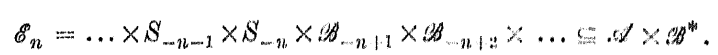

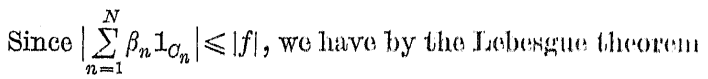

$$
\mathbb{E}^{\mathscr{s}} m f=\sum_{n=1}^{\infty} \beta_{n} \mathbb{E}^{\infty} m 1_{O_{n}} \quad \text { for } \quad m \geqslant 0
$$

Observe that

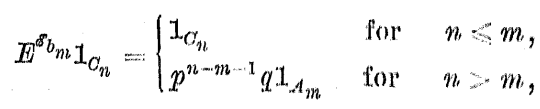

for $m \geqslant 0$. Using (8), (9) and (4) wo obtain

$$
E^{g_{b}} f=\sum_{n=1}^{m} \beta_{n} 1_{C_{n}}+a_{m} 1_{A_{m}} \quad \text { for } \quad m \geqslant 0
$$

Clearly,

(11) $\quad E^{8 b_{m}+k} f=E^{8 b_{m}} \quad$ for $\quad k=0,1, \ldots, b_{m+1}-b_{m}-1, m \geq 0$.

Now, denote $D_{k}=\left\{E^{s^{g}} f \geqslant a_{k}\right\}\left(l_{0} \cdots 0,1,2, \ldots\right)$. Wo shall prove that

$$
D_{l k}=A_{m} \quad \text { for } \quad b_{m} \leqslant k_{k} \leqslant b_{m+1}-1, m=0 .
$$

Fix $m \geqslant 0$ and $b_{m} \leqslant k \leqslant b_{m+1} \cdots 1$. Using (1.1), (10) and the monotonicity of $a_{n}$, we have

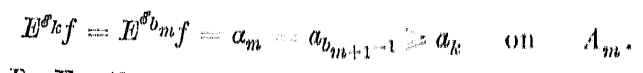

Thus $A_{m} \subseteq D_{k}$. For the proof of the converse inclusion observes that

(13) $\quad \beta_{n}=q^{-1}\left(\alpha_{n-1}-p a_{n}\right)<a_{n-1}=a_{b_{n-1}}<a_{b_{n}}$ fior $n \geqslant 1$.

Using (11), (10) and (13), wo obtain.

$$
E^{\delta_{k f}}=\mathbb{E}^{\delta^{b_{m}}} f=\sum_{i=1}^{m} \beta_{i} \mathcal{I}_{C_{i}} \leqslant \max _{i=1, \ldots, m} \beta_{i}<a_{b_{m}} \leqslant a_{k} \quad \text { on } \quad A_{m}^{a}
$$

Thus $A_{m}^{c} \subseteq D_{k}^{c}$, which proves (12). (The case $m=0$ in the last inclusion is trivial.)

It follows from (12) that

$$
\begin{aligned}
\sum_{k_{n=0}}^{\infty}\left(\mu \times \lambda^{*}\right)\left(D_{l c}\right) & =\sum_{n=0}^{\infty}\left(b_{n+1}-b_{n}\right)\left(\mu \times \lambda^{*}\right)\left(A_{n}\right) \\
& =\sum_{n=0}^{\infty}\left(b_{n+1}-b_{n}\right) p^{n} .
\end{aligned}
$$

However, we infer from (6) that there exists $\varepsilon>0$ such that $b_{n+1}-b_{n}$ $\geqslant \varepsilon b_{n}$ for $n \geqslant 0$. Thus (1.4) and (5) imply

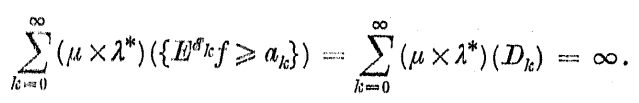

Denoto $F_{n}^{\prime}=\left\{E^{r^{n}} U^{n} f \geqslant a_{n}\right\}$. It is easy to check that $E^{\mathscr{8}} U^{n}=U^{n} E^{\mathbb{S}_{n}}$ for $n \geqslant 0$. Thus $F_{n}=\left\{U^{n} \mathbb{E}^{\delta_{n}} f \geqslant a_{n}\right\}=p^{*-n} D_{n}$. Since $\varphi^{*}$ is measurepresorving, (15) implies

$$
\sum_{n=0}^{\infty}\left(\mu \times \lambda^{*}\right)\left(F_{n}\right)=\infty
$$

Wo shall noed the following

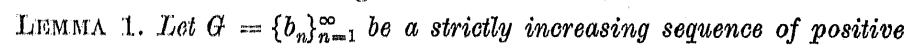
integers and lot $\psi: N \rightarrow N$ be the shift transformation on positive integers. Denote by $\psi^{n} G$ the image of $G$ under $\psi^{n}$ and by $|\cdot|$ the number of elements of a given set. If the sequence $G=\left\{b_{n}\right\}_{n=1}^{\infty}$ satisfies the Hadamard gap condition, i.e. if there exists an $\varepsilon>0$ such that

then

$$
\frac{b_{n+1}}{b_{n}} \geqslant 1+\varepsilon \quad \text { for } \quad n=1,2, \ldots
$$

$$
\operatorname{sip}_{n \rightarrow 1}\left|G \cap \psi^{n} G\right|<2-\frac{\log \varepsilon}{\log (1+\varepsilon)}<\infty .
$$

1'roof. Suppose that for some $n \geqslant 1$ and $N \geqslant 1$ we have

$$
b_{l_{i}}+n=b_{l_{i}} \quad(i=1,2, \ldots, N),
$$

where $l_{1}<l_{2}<\ldots<l_{N}$. Denote $x=b_{l_{N-1}}$. Since $l_{N}>k_{N}$, we have $b_{l_{N}-1} \geq b_{l_{N}}$. Thus, using (18) and (17) we obtain $b_{l_{1}}-b_{k_{1}}=n=b_{l_{N}}-$ $-b_{l_{l_{N}}} \geqslant b_{l_{N}}-b_{l_{N^{-1}}} \geqslant \varepsilon b_{l_{N^{-1}}}=\varepsilon x$. In particular,

$$
b_{l_{1}}>\varepsilon x \text {. }
$$

Using (17) and (19) we have $b_{l_{k+1}} \geqslant b_{l_{1}+k} \geqslant b_{l_{1}}(1+\varepsilon)^{k}>\varepsilon(1+\varepsilon)^{k} x$ for 
$k=0,1, \ldots, N-1$. Hence $b_{l_{N-1}}>\varepsilon(1+\varepsilon)^{N-2} x$. On the other hand $b_{l_{N-1}} \leqslant b_{l_{N-1}}=x$. Therefore $\varepsilon(1+\varepsilon)^{N-2}<1$, i.o.

$$
N-2<-\frac{\log \varepsilon}{\log (1+\varepsilon)}
$$

which proves the lomma.

Using (12) it is easy to check that

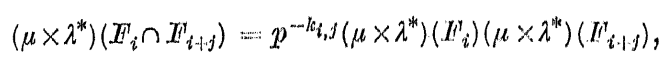

where $k_{i, j}=\left|G \cap \psi^{j} G \cap\{1,2, \ldots, i+j\}\right|, G=\left\{b_{1}, b_{2}, \ldots\right\}, j \geq 1, i \geqslant 0$. Thus (6) and Lemma 1 imply that there exists an $K<\infty$ such that

$$
\left(\mu \times \lambda^{*}\right)\left(H_{m}^{\prime} \cap H_{n}^{\prime}\right) \leqslant K\left(\mu \times \lambda^{*}\right)\left(H_{m}^{\prime}\right)\left(\mu \times \lambda^{*}\right)\left(H_{n}^{\prime}\right)
$$

for all $m, n \geqslant 0, m \neq n$.

We have

Lencra 2 (see [9]). Let $(\Omega, \mathscr{F}, P)$ be a probability space and let $\left\{T_{n}^{\prime}\right\}_{n=1}^{\infty}$ be a sequence of events satisfying $\sum_{n=1}^{\infty} P\left(F_{n}\right)=: \infty$. If for some constant $K<\infty$ and for all $m$ and $n$ with $m \neq n$ we have $P\left(T_{m} \cap H_{n}^{\prime}\right) \leqslant K P\left(T_{m}\right) P\left(F_{n}\right)$, then $P\left(F_{n}\right.$ i.o. $)>0$.

Now, (16), (20) and Lemma 2 imply that $\left(\mu \times \lambda^{*}\right)\left(F_{n}\right.$ i.o.) $)$ o. Finully, we shall use the following

Lewara 3 (see [16], Corollary 2 and the footnotio on p. 140). Let $(\Omega, \mathscr{F}, P, T)$ be an ergodio dynamical systom. If $\left\{D_{n}\right\}_{n=1}^{\infty}$ is a non-inoreasing sequence of events, then $P\left(T^{-n} D_{n}\right.$ i.o. $)=0$ or 1 .

Since $F_{n}=\varphi^{*-n} D_{n}$ and, by (12), $D_{n}$ is a non-incrensing sequence of sets we can apply Lemma 3 which gives $\left(\mu \times \lambda^{*}\right)\left(N_{n}^{*}\right.$ i.o. $)=1$. This proves (7).

Now, let $a_{n}=(n+2) \log (n+2) \quad(n=0,1,2, \ldots)$ and lot

$$
b_{0}=0, \quad b_{n}=\left[\frac{1}{p^{N+n}(N+n) \log (N+n)}\right]-1 . \quad(n-1,2, \ldots),
$$

where $[x]$ denotes the integral part of $x$. Trere $N$ is an nutum number so large that $b_{n}$ is strictly increasing. Wo huve

$$
\lim _{n \rightarrow \infty} \frac{b_{n+1}}{b_{n}}=p^{-1}>1
$$

which gives (6). Noxt, it is easy to eheck that (1) and (5) loold. Now, wo shall verify (3). Since

$$
\frac{1}{p^{n} n \log n}-1 \geqslant \frac{1}{p^{n-1 / n} n \log n}
$$

for sufficiontly large $n$ and since the function

$$
h(x)=\frac{1}{x \log x} \log \frac{1}{p^{x} x \log x}
$$

is decreasing for sufficiently large $x$, we have

$$
\begin{aligned}
& {\left[\frac{1}{p^{n} n \log n}\right] \log \left[\frac{1}{p^{n} n \log n}\right]-p\left[\frac{1}{p^{n+1}(n+1) \log (n+1)}\right] \times} \\
& \times \log \left[\frac{1}{p^{n+1}(n+1) \log (n+1)}\right] \geqslant\left(\frac{1}{p^{n} n \log n}-1\right) \log \frac{1}{p^{n-1 / n} n \log n}- \\
& \quad-\frac{1}{p^{n} n \log n} \log \frac{1}{p^{n} n \log n}=-\frac{\log (1 / p)}{p^{n} n^{2} \log n}-\log \frac{1}{p^{n-1 / n} n \log n}
\end{aligned}
$$

for sufficientily large $n$ Since.

$$
\sum_{n}\left(\frac{\log 1 / p}{p^{n} n^{2} \log n}+\log \frac{1}{p^{n-1 / n} n \log n}\right) p^{n-N}<\infty,
$$

we obtain (3). Therefore we have

$$
\left(\mu \times \lambda^{*}\right)\left(\left\{\mathbb{E}^{\delta} U^{n} f \geqslant n \log n \text { i.o. }\right\}\right)
$$

$$
\geqslant\left(\mu \times \lambda^{*}\right)\left(\left\{B^{8} U^{n} f \geqslant(n+2) \log (n+2) \text { i.o. }\right\}\right)=1 .
$$

Taking $|f|$ instead of $f$, we obtain (2) .

\section{References}

[1] D. I. Burkholder, Successive conditional expectations of an integrable function, Ann. Matil. Statist. 33 (1962), 887-893.

[2] R. Cairoli, Sur le théorème ergodique aléatoire, Bull. Sci. Math. 88 (1964), 31-37.

[3] S. D. Chatterji, Martingale convergence and the Radon-Nitodym theorem in Bincath spuose, Math. Scand. 22 (1968), 21-41.

[.] N. Dunford and J. T. Schwartz, Linear Operators, Part I, Interscience, Now York 1958.

[5] S. R. Foguol, The Zirgodio Theory of Markov Processes, Van Nostrand Reinhold Go., Now York 1969.

[6] S. Gladysz, Lin ergodischer Satz, Studia Math. 15 (1956), 148-157.

[7] - Ubor den stochastischen Ergoclensatz, ibid. 15 (1956), 158-173.

[8] \$. Kalcutani, Random ergodic theorems and Markoff processes with a stable distribution, Proc. 2nd Barkeley Symp. Math. Statist. Prob., 1951, 247-261.

[9] J. Lamperti, Wiener's test and Markov ohains, J. Math. Anal. Appl. 6 (1963), $58-60$.

[10] P. A. Moyor, Probability and Potentials, Blaisdell, 1966.

[11] K. N a, wrotzki, Ein zufülliger Ergodensatz für eine Familie stoohastisoher Matrizen 
ohne gemeinsames invariantes Verteilungsgesetz, Matih. Nachir. 70 (1975), 15-28. [12] J. Neveu, Discrete-Parameter Martingales, North-Holland, Amstordam 1975.

[13] - Mathematical Froundations of the Oalculus of Probability, ITolden-Day, San Francisco 1965.

[14] D. Rovuz, Marloov Ohains, Nortih-Molland, Amstiordam 1975.

[15] C. Ryll-Nardzowski, On the ergodio theorems (III) (T'he rathdom ergodio theorem), Studia Matil. 14 (1954), 298-301.

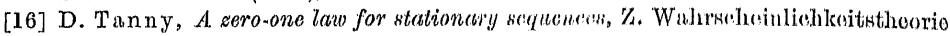
verw. Geb. 30 (1974), 139-148.

[17] J. Woś, Random orgodio theorem for Dunfordmsohumarts oprarabors, Bull. Acad. Polon. Sci. 27 (1979), 806 -867.

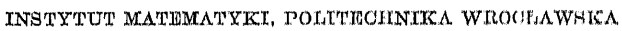

Wybrzezo Wyspiarakiego $27,50 * 370$ Wroclaw

Reoeived Maroh 20, 1979

Revised version May 14, 1981 